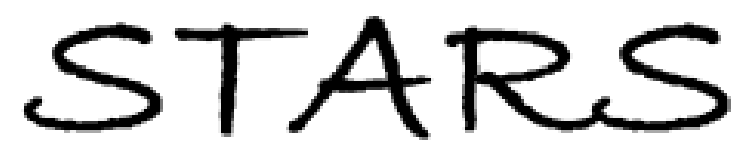

University of Central Florida

STARS

$1-1-2012$

\title{
Computational methodology for analysis of the Soret effect in crystals: Application to hydrogen in palladium
}

Patrick K. Schelling

University of Central Florida

Timothy Le

University of Central Florida

Find similar works at: https://stars.library.ucf.edu/facultybib2010

University of Central Florida Libraries http://library.ucf.edu

This Article is brought to you for free and open access by the Faculty Bibliography at STARS. It has been accepted for inclusion in Faculty Bibliography 2010 s by an authorized administrator of STARS. For more information, please contact STARS@ucf.edu.

\section{Recommended Citation}

Schelling, Patrick K. and Le, Timothy, "Computational methodology for analysis of the Soret effect in crystals: Application to hydrogen in palladium" (2012). Faculty Bibliography 2010s. 3261.

https://stars.library.ucf.edu/facultybib2010/3261

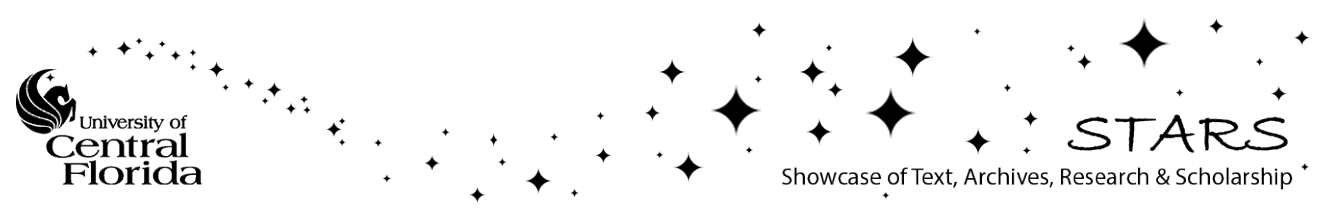




\section{Computational methodology for analysis of the Soret effect in crystals: Application to hydrogen in palladium}

Cite as: J. Appl. Phys. 112, 083516 (2012); https://doi.org/10.1063/1.4758462

Submitted: 01 July 2012 . Accepted: 12 September 2012 . Published Online: 22 October 2012

Patrick K. Schelling, and Timothy Le

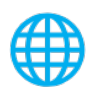

View Online

\section{ARTICLES YOU MAY BE INTERESTED IN}

Atomic-scale simulation of the thermodiffusion of hydrogen in palladium

Journal of Applied Physics 114, 063509 (2013); https://doi.org/10.1063/1.4816961

Analysis of simulation methodology for calculation of the heat of transport for vacancy thermodiffusion

Journal of Applied Physics 116, 023504 (2014); https://doi.org/10.1063/1.4887121

The Statistical Mechanical Theory of Transport Processes. IV. The Equations of Hydrodynamics The Journal of Chemical Physics 18, 817 (1950); https://doi.org/10.1063/1.1747782

Applied Physics Reviews Now accepting original research

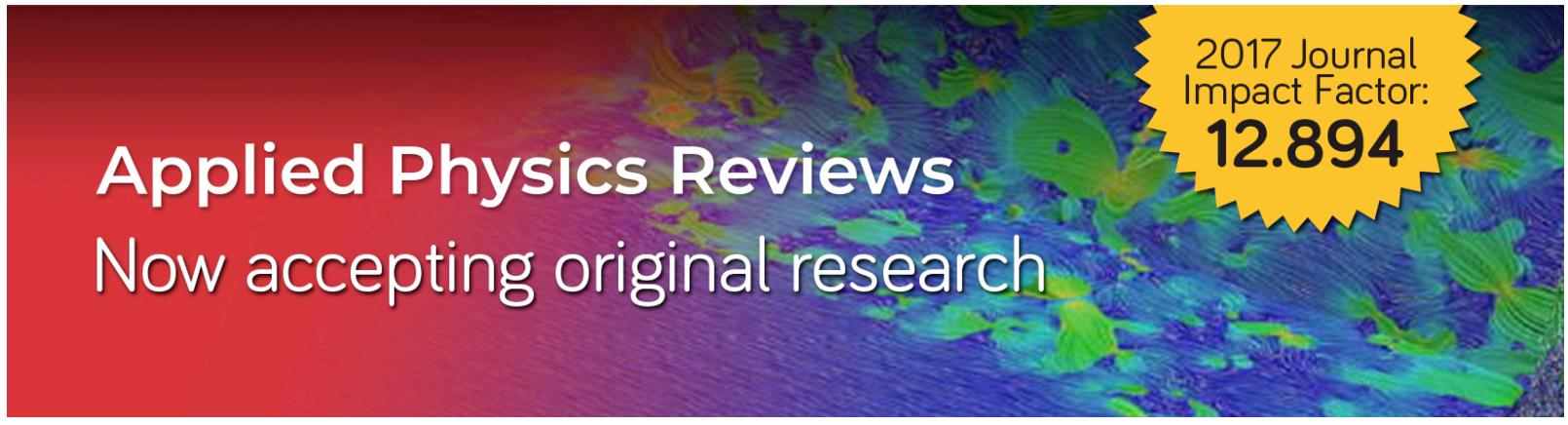




\title{
Computational methodology for analysis of the Soret effect in crystals: Application to hydrogen in palladium
}

\author{
Patrick K. Schelling and Timothy Le \\ Advanced Material Processing and Analysis Center, Department of Physics University of Central Florida, \\ 4000 Central Florida Blvd. Orlando, Florida 32816, USA
}

(Received 1 July 2012; accepted 12 September 2012; published online 22 October 2012)

\begin{abstract}
Different computational methodologies to compute thermodiffusion of hydrogen in palladium are explored. It is found that diffusion occurs rapidly enough to directly observe thermodiffusion in the presence of an applied temperature gradient. This provides an unequivocal result that hydrogen moves from low to high temperatures, corresponding to a negative value for the reduced heat of transport $Q^{* \prime} \approx-0.3 \mathrm{eV}$, which can be used to validate other methods. Further simulations using the Green-Kubo formulae and a recently developed constrained-dynamics approach are found to be in agreement with direct simulation results. In particular, in each of the three methods used, the value of $Q^{* \prime}$ is found to be in the range between -0.3 and $-0.2 \mathrm{eV}$. We show how to correctly define and compute the partial-enthalpy term for hydrogen which is key to obtaining accurate results. The results provide important foundational and numerical validation for the constrained-dynamics approach. The advantage of the constrained-dynamics method is that it can be applied to thermodiffusion in materials where diffusion does not occur on a molecular-dynamics time scale. Finally, we show that the empirical potential predicts behavior that is not in agreement with experiment. In particular, experiments are reported to show hydrogen diffusing from high to low temperatures corresponding to a positive value for $Q^{* \prime}$. (C) 2012 American Institute of Physics. [http://dx.doi.org/10.1063/1.4758462]
\end{abstract}

\section{INTRODUCTION}

Thermodiffusion is the phenomenon of mass transport in response to a temperature gradient. Alternately, it refers to the heat flux that accompanies a mass flow. In solid crystalline materials, this effect has been the focus of several theoretical and experimental studies over a period of several decades. Yet progress in developing fundamental understanding has been quite slow, and there is still little known especially at the atomic scale. For example, in a two component system such as hydrogen in palladium with an applied temperature gradient, there are few simple arguments that have been tested which can predict even the direction of the flow of hydrogen. Moreover, a simple picture that explains the magnitude of the driving force for thermodiffusion is lacking. Simple kinetic theory has suggested that the key parameter that describes the thermodiffusion driving force, the reduced heat of transport $Q^{* \prime}$ (see Sec. II for a definition), should be comparable to the activation energy $\Delta U_{a c t}$ for migration. ${ }^{1,2}$ However, experimental observations do not support this simple picture, and in many cases $Q^{* \prime}$ is found to be either much smaller or much larger than $\Delta U_{\text {act }{ }^{1}}{ }^{1}$

Atomic scale simulation using simple empirical potentials can play a key role in answering these questions and hence provide a basis for a fundamental theory of thermotransport. However, while there have been many efforts using molecular-dynamics (MD) simulation, a clear picture is yet to emerge. One of the primary obstacles is the fact that coupled heat and mass transport is a relatively weak effect in comparison to ordinary isothermal diffusion and conductive thermal transport. Moreover, in most instances of practical importance, $\Delta U_{a c t}$ is significantly greater than $k_{B} T$, and diffusion does not occur on a typical MD time scale. Consequently, direct brute-force simulation is generally impractical.

Several techniques exist for determining $Q^{* \prime}$ from MD simulation. The Green-Kubo method ${ }^{3,4}$ uses time-correlation functions of the coupled heat an mass fluxes to compute transport coefficients. This approach was used by Gillan, 5,6 who was able to demonstrate practicality for the Pd:H system. However, $\mathrm{Pd}: \mathrm{H}$ is a special case because hydrogen diffuses quite rapidly. In cases where ordinary diffusion does not occur on an MD time scale, Green-Kubo methods are not practical. Gillan also explored applying external forces ${ }^{7}$ to drive heat and mass currents, which was again found to provide reasonable results. In particular, a value of $Q^{* \prime}$ was obtained from the heat flux that results from a driven hydrogen mass flux. ${ }^{6}$ Recently, we have developed a technique, referred to hereafter as the constrained-dynamics approach, which can be used to determine the driving force for thermodiffusion by computing the work required to drive an atom through the transition state in the presence of an applied temperature gradient. ${ }^{8}$ The practical advantage of constrained dynamics is that it can be used to compute transport parameters in problems where the underlying hopping events are too slow to be observed on an MD time scale. This includes, for example, vacancy-mediated thermodiffusion in singlecomponent or binary-alloy metal. While the results are promising, as of now there are no published results that validate the methodology. In part this can be accomplished by direct comparison to simulation results using established approaches. There is also a need to put constraineddynamics simulation on a more solid theoretical foundation. Finally, several numerical aspects of the approach should be explored, including finite-size and nonlinear effects. 
In this article, we simulate thermodiffusion of hydrogen in a palladium lattice. The primary objective is to more firmly establish the theoretical foundation of the constrained-dynamics method first presented in Ref. 8, and also to establish if predictions using this approach are in agreement with other methods. Because hydrogen diffuses rapidly in the system, we find that "direct" simulation of thermodiffusion is possible by computing the average drift of hydrogen in the presence of a temperature gradient. An approach for estimating $Q^{* \prime}$ based on the direct observation of hydrogen drift is outlined. We revisit the Green-Kubo calculations first performed by Gillan for the Pd:H system. ${ }^{5,6}$ We show that a correct definition of the partial enthalpy is key to obtaining correct results using Green-Kubo methods. Finally, results using the constrained-dynamics method are reported. The three techniques are found to give consistent results for $Q^{* \prime}$. Specifically, $Q^{* \prime}$ is found to be negative indicating that hydrogen tends to drift in the direction of increasing temperature. In summary, the results presented here demonstrate that the constrained-dynamics approach in Ref. 8 provides a reliable method to compute $Q^{* \prime}$. This should be of use for more challenging cases where Green-Kubo and direct methods are not practical.

\section{THEORETICAL BACKGROUND}

In this article, we are primarily interested in the coupled transport of heat and matter in the two component Pd:H system. We define the mass flux $\vec{J}_{1}$ for Pd,

$$
\vec{J}_{1}=\sum_{i=1}^{N_{1}} \vec{v}_{i}
$$

where the sum is only over the $N_{1} \mathrm{Pd}$ atoms. Similarly, the mass flux for the hydrogen atoms is,

$$
\vec{J}_{2}=\sum_{i=1}^{N_{2}} \vec{v}_{i}
$$

where the sum is only over the $N_{2}$ hydrogen atoms. Both of the mass fluxes $\vec{J}_{1}$ and $\vec{J}_{2}$ are defined relative to the centerof-mass reference frame. Hence, there is clearly a relation between the mass fluxes, in particular,

$$
m_{1} \vec{J}_{1}+m_{2} \vec{J}_{2}=0,
$$

where $m_{1}$ is the mass of $\mathrm{Pd}$ and $m_{2}$ is the mass of hydrogen. Because $m_{2} \ll m_{1}$, the flux of hydrogen $\vec{J}_{2}$ is dominant. Hence, it is sufficient to consider only the hydrogen flux $\vec{J}_{2}$ and energy flux $\vec{J}_{q}$. In the section describing Green-Kubo calculations, explicit expressions for the energy flux $\vec{J}_{q}$ and heat flux $\vec{J}_{q}^{\prime}$ for the case of a pair potential will be given.

The phenomenological transport equations for mass and energy flux are ${ }^{1}$

$$
\begin{aligned}
& \vec{J}_{2}=\frac{\Omega}{k_{B} T}\left[L_{22} \vec{X}_{2}+L_{2 q} \vec{X}_{q}\right], \\
& \vec{J}_{q}=\frac{\Omega}{k_{B} T}\left[L_{q 2} \vec{X}_{2}+L_{q q} \vec{X}_{q}\right],
\end{aligned}
$$

where $\Omega$ is the system volume. The Onsager reciprocal relations ${ }^{9}$ assert that

$$
L_{q 2}=L_{2 q} .
$$

The driving forces are defined by

$$
\begin{gathered}
\vec{X}_{2}=\vec{F}_{e x t}-T \vec{\nabla}\left(\frac{\mu_{2}}{T}\right), \\
\vec{X}_{q}=-\frac{1}{T} \vec{\nabla} T,
\end{gathered}
$$

where $\vec{F}_{\text {ext }}$ is an external force applied to the hydrogen atoms and $\mu_{2}$ is the chemical potential of hydrogen.

The phenomenological equations can be written for alternative definitions of the driving forces and fluxes. An alternate definition for the thermodynamic driving forces is

$$
\begin{gathered}
\vec{X}_{2}^{\prime}=\vec{X}_{2}+h_{2} \vec{X}_{q}, \\
\vec{X}_{q}^{\prime}=\vec{X}_{q},
\end{gathered}
$$

with corresponding fluxes given by

$$
\begin{gathered}
\vec{J}_{2}^{\prime}=\vec{J}_{2}, \\
\vec{J}_{q}^{\prime}=\vec{J}_{q}-h_{2} \vec{J}_{2} .
\end{gathered}
$$

Here, $h_{2}$ refers to the partial enthalpy of hydrogen in the Pd lattice. The transport equations are defined identically as Eqs. (4) and (5)

$$
\begin{aligned}
& J_{2}^{\prime}=\frac{\Omega}{k_{B} T}\left[L_{22}^{\prime} \vec{X}_{2}^{\prime}+L_{2 q}^{\prime} \vec{X}_{q}^{\prime}\right], \\
& \vec{J}_{q}^{\prime}=\frac{\Omega}{k_{B} T}\left[L_{q 2}^{\prime} \vec{X}_{2}^{\prime}+L_{q q}^{\prime} \vec{X}_{q}^{\prime}\right] .
\end{aligned}
$$

The transport coefficients for the two descriptions are related by

$$
\begin{gathered}
L_{22}^{\prime}=L_{22}, \\
L_{2 q}^{\prime}=L_{2 q}-h_{2} L_{22}, \\
L_{q q}^{\prime}=L_{q q}-2 h_{2} L_{2 q}+h_{2}^{2} L_{22} .
\end{gathered}
$$

We next define the heat of $\operatorname{transport}^{1} Q^{*}$ using

$$
L_{2 q}=L_{q 2} \equiv Q^{*} L_{22}
$$

Using this definition and also Eq. (16), we also see that

$$
L_{2 q}^{\prime}=\left(Q^{*}-h_{2}\right) L_{22}=Q^{* \prime} L_{22},
$$

where the quantity $Q^{* \prime}$ is most often called the reduced heat of transport.

In the present study, the definitions in Eqs. (13) and (14) are most convenient. We use the definition of the $Q^{* \prime}$ and find 


$$
\vec{J}_{2}=-\frac{L_{22} \Omega}{k_{B} T}\left(\vec{\nabla} \mu_{2}\right)_{T}+\frac{L_{22} \Omega}{k_{B} T}\left[\vec{F}_{e x t}-Q^{* *} \frac{\vec{\nabla} T}{T}\right],
$$

where we have used that $\vec{J}_{2}^{\prime}=\vec{J}_{2}$ and $L_{22}^{\prime}=L_{22}$. The gradient term $\left(\vec{\nabla} \mu_{2}\right)_{T}$ represents the gradient of the hydrogen chemical potential due to concentration and pressure gradients only. In the following, we refer exclusively to Eq. (20) for the description of the mass flux $\vec{J}_{2}$. In the absence of any pressure or concentration gradient, the hydrogen flux is

$$
\vec{J}_{2}=\frac{L_{22} \Omega}{k_{B} T}\left[\vec{F}_{e x t}-Q^{* \prime} \frac{\vec{\nabla} T}{T}\right] .
$$

This expression clearly demonstrates that the driving force for thermodiffusion is given by $\vec{F}_{e x t}-Q^{* \prime} \frac{\vec{\nabla} T}{T}$. This force is relevant, for example, for the driving force acting on a single hydrogen atom in Pd with an imposed temperature gradient and externally applied force.

\section{SIMULATION APPROACH}

In the MD simulations, we use the empirical pair potentials reported in Ref. 5. The interaction between palladium atoms is given by a sixth-order polynomial

$$
V_{P d-P d}(r)=\sum_{s=1}^{6} a_{s} r^{s}
$$

For the interaction between hydrogen atoms, the pair potential is taken to be the same as that which describes the Pd-H interaction

$$
V_{P d-H}(r)=V_{H-H}(r)=A \exp (-r / \rho) .
$$

The parameters and cutoff distances $r_{c}$ for the interactions are given in Ref. 5. In preliminary MD simulations, small discontinuities in the total energy were observed due to the discontinuity of the potential energy and resulting forces at the cutoff distance $r_{c}$. We remedied this using a standard procedure to remove discontinuities in the potential energy and forces. In particular, we shift each potential and obtain the smooth potential $V^{\prime}(r)$ from

$$
V_{\alpha \beta}^{\prime}(r)=V_{\alpha \beta}(r)-V_{\alpha \beta}\left(r=r_{c}\right)-\left(r-r_{c}\right)\left[\frac{d V_{\alpha \beta}}{d r}\right]_{r=r_{c}},
$$

where $\alpha \beta$ represents the particular pair, $\mathrm{Pd}-\mathrm{Pd}, \mathrm{Pd}-\mathrm{H}$, or $\mathrm{H}-\mathrm{H}$. In this way, the potentials $V_{\alpha \beta}^{\prime}(r)$ and the first derivatives $\frac{d V_{\alpha \beta}}{d r}$ smoothly approach zero at the cutoff distance $r_{c}$. It was initially thought that this procedure would simply remove the discontinuities without resulting in significant changes in the interactions. However, it was found that some noticeable quantitative differences occurred. However, the shape of the potential functions was only slightly altered, and the main effect was a shift in energy. For example, the energy required to insert a single hydrogen into the Pd lattice at a fixed lattice parameter $a_{0}=4.07 \AA$ and $T=0^{\circ} \mathrm{K}$ was $1.32 \mathrm{eV}$. This is compared to the value reported in Ref. 5 of $1.61 \mathrm{eV}$. This downward shift of $0.29 \mathrm{eV}$ is due in part to a constant shift in the repulsive $\mathrm{Pd}-\mathrm{H}$ potential, and also a small constant shift in the Pd-H repulsive force. We show below, however, that the overall description of thermodiffusion was not significantly affected by the smoothing procedure. In particular, differences in the Pd-H interaction approximately cancel in the calculation of the reduced heat of transport $Q^{* \prime}$.

The first requirement for the direct simulation of thermodiffusion is that diffusion occurs rapidly on an MD time scale. In all the calculations reported here, the MD time step was $\Delta t=0.414 f s$. We first simulate diffusion in isothermal conditions using a system with $N_{1}=2048 \mathrm{Pd}$ atoms and $N_{2}=10$ hydrogen atoms. We obtain the diffusion coefficient $D$ from the mean-squared displacement $\left\langle\Delta r^{2}\right\rangle$ using the Einstein relation

$$
6 D \tau=\left\langle\Delta r^{2}\right\rangle_{\tau}
$$

where $\tau$ is the simulation time for which the average $\left\langle\Delta r^{2}\right\rangle_{\tau}$ was computed. We performed four independent simulations each of duration 44.9 ps to obtain $D$ at $T=980^{\circ} \mathrm{K}$. We found $D=3.03 \times 10^{-4} \mathrm{~cm}^{2} / \mathrm{s}$. Simulations over a range from $T=680^{\circ} \mathrm{K}$ to $T=1280^{\circ} \mathrm{K}$ were used to generate an Arrhenius plot, from which we determined the activation energy $\Delta U_{a c t}=0.25 \mathrm{eV}$ for migration. This is to be compared to $D=2.23 \times 10^{-4} \mathrm{~cm}^{2} / \mathrm{s}$ at $T=980^{\circ} \mathrm{K}$ and $\Delta U_{a c t}=0.29 \mathrm{eV}$ from the fit in Ref. 5. Thus, while the results are not too different, the potentials used here with the discontinuities smoothed out result in slightly different selfdiffusion behavior. From these results, we see that on average about $40-50$ hops occur within 40 ps of simulation time. This quite fast diffusion rate, as we will see in Secs. IV and V, is sufficient to observe thermodiffusion effects on an MD time scale.

The simulation cell used in direct thermodiffusion simulations and constrained-dynamics simulations was a rectangular prism. We define a Cartesian coordinate system coincident with the vectors that define the supercell. The $\mathrm{x}$-, $\mathrm{y}-$, and $\mathrm{z}$ - directions were chosen to be coincident with the [110], [001], and [110] crystallographic directions, respectively. For this supercell, it is convenient to define a twoatom unit cell. The supercell then consisted of $8 \times 6 \times 28$ unit cells with a total of $2688 \mathrm{Pd}$ atoms in an fcc lattice. To simulate non-equilibrium conditions with an applied temperature gradient $\vec{\nabla} T$, we use the method developed by Jund and Jullien in Ref. 10. In this method, two thin regions are defined where heating and cooling takes place. The thickness of the hot and cold reservoirs was taken to be $a_{0} / \sqrt{2}$, with $a_{0}=4.07 \AA$. A single hydrogen atom is added in an octahedral interstitial site approximately equidistant from the hot and cold reservoirs. In the hot region, we add an energy $\Delta \epsilon=0.01 \mathrm{eV}$ at each MD time step by rescaling the velocity of the atoms in the hot reservoir. The same amount of energy is removed at each MD step in the cold reservoir. Given these parameters, we obtain $J_{q}^{\prime} / \Omega=2.15 \times 10^{11} \mathrm{~W} / \mathrm{m}^{2}$ for the input heat current.

For Green Kubo calculations, we use a small cell with $N_{1}=108 \mathrm{Pd}$ atoms and $N_{2}=20$ hydrogen atoms. In the equations and simulations reported here, the mass and heat 
flux were computed in a center of mass reference frame. Because the mass of Pd is much greater than that of hydrogen, there is little difference between a reference frame fixed to the lattice, as was done in Ref. 6, and the center-of-mass reference frame.

\section{RESULTS}

\section{A. Direct thermodiffusion simulation}

We first consider direct simulation of thermodiffusion by applying a temperature gradient in the $8 \times 6 \times 28$ cell with $2688 \mathrm{Pd}$ atoms and a single hydrogen atom described in Sec. III. After establishing equilibrium at $T=980^{\circ} \mathrm{K}$, a temperature gradient was established over $9000 \mathrm{MD}$ steps or 3.73 ps. During the first 3.31 ps the hydrogen atom was held fixed in place, and then it was allowed to freely diffuse. The displacement of the hydrogen atom was determined as a function of time over 20.72 ps. In Fig. 1, the time-averaged temperature gradient is shown. In Fig. 2, we show the average displacement of the hydrogen atom $\left\langle\Delta r_{x}\right\rangle,\left\langle\Delta r_{y}\right\rangle$, and $\left\langle\Delta r_{z}\right\rangle$ in the three Cartesian directions averaged over 60 independent simulations. The results show that the hydrogen atom drifts toward the hot reservoir by a distance $\left\langle\Delta r_{z}\right\rangle=-097 a_{0}$.

The result in Eq. (21) shows that computing the current $\vec{J}_{2}$ in the presence of a temperature gradient $\vec{\nabla} T$ can be used to determine the reduced heat of transport $Q^{* \prime}$. We first analyze the results in Fig. 2 to provide an estimate for $\vec{J}_{2}$ in the case where $\vec{F}_{\text {ext }}=0$. We consider the averaged quantity $\left\langle\vec{J}_{2} \cdot \hat{k}\right\rangle$ where $\hat{k}$ is a unit vector along the $\mathrm{z}$-direction of the simulation cell. Assuming a uniform average drift velocity, we obtain

$$
\left\langle\vec{J}_{2} \cdot \hat{k}\right\rangle=\frac{\left\langle\Delta r_{z}\right\rangle}{\tau} \approx-\frac{0.97 a_{0}}{\tau} .
$$

Then, using Eq. (21) with $\vec{F}_{\text {ext }}=0$, we obtain a prediction for $Q^{* \prime}$,

$$
Q^{* \prime}=-\left[\frac{k_{B} T^{2}}{L_{22} \Omega}\right] \frac{\left\langle\vec{J}_{2} \cdot \hat{k}\right\rangle}{\langle\vec{\nabla} T \cdot \hat{k}\rangle} .
$$

The time-averaged temperature gradient is estimated from the data shown in Fig. 1 to be $-15.8^{\circ} \mathrm{K} \AA^{-1}$. To determine the

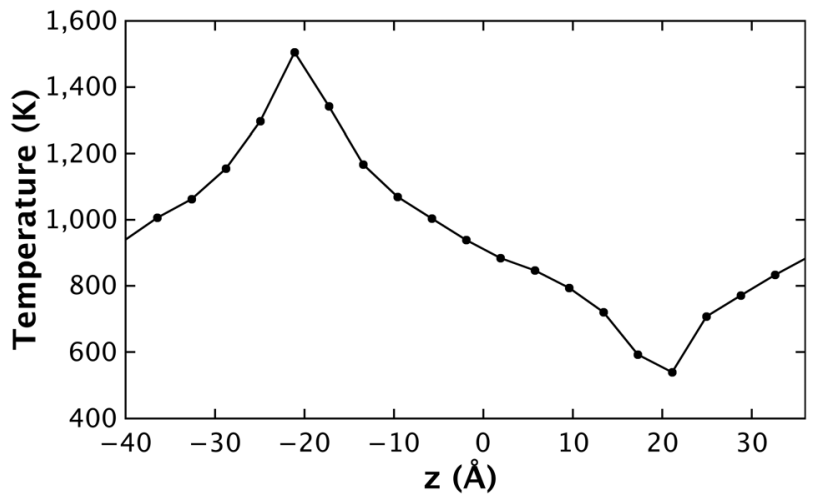

FIG. 1. Time-averaged temperature profile computed for direct simulations of thermodiffusion. The temperature profiles for constrained-dynamics simulations are essentially identical.

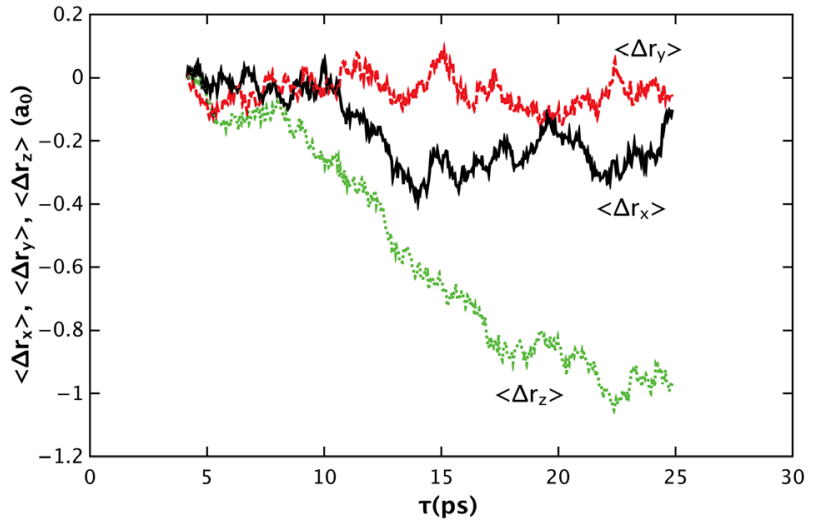

FIG. 2. Average displacements $\left\langle\Delta r_{x}\right\rangle$ (black solid line), $\left\langle\Delta r_{y}\right\rangle$ (red dashed line), and $\left\langle\Delta r_{z}\right\rangle$ (green dotted line) in the presence of a temperature gradient with $\vec{F}_{\text {ext }}=0$ in units of the lattice parameter $a_{0}=4.07 \AA$. The results show a clear drift of the hydrogen atom in the negative $z$-directions, hence towards higher temperatures.

magnitude of the temperature gradient, only the linear portion of the temperature profile at least $12 \AA$ away from the hot and cold reservoirs was used. For a single hydrogen atom in a volume $\Omega$, we used that $L_{22} \Omega=D$ where $D=3.03 \times 10^{-4} \mathrm{~cm}^{2} / \mathrm{s}$ is the diffusion coefficient computed in Sec. III. Combining, the expression above gives $Q^{* \prime}=-0.33 \pm 0.10 \mathrm{eV}$. The estimate for the error comes from the apparent error of $\pm 0.30 a_{0}$ in the determination of $\left\langle\Delta r_{z}\right\rangle$ due to random diffusion. The negative sign of $Q^{* \prime}$ is consistent with the tendency of the hydrogen atom to drift towards the hot side of the simulation cell. This result is in contradiction to the Green-Kubo results reported by Gillan. ${ }^{6}$ Later in this section, we will address this apparently contradictory result.

Equation (21) indicates that it should be possible to apply an external force $\vec{F}_{\text {ext }}$ to cancel the driving force due to the temperature gradient and hence provide another approach to estimate $Q^{* \prime}$. This is analogous to the thermoelectric effect, where the Seebeck coefficient is determined by measuring the electrostatic potential that exactly balances the driving force due to an imposed temperature gradient. We define $\vec{F}_{\text {ext }}^{0}$ to be the external force determined to result in $\left\langle\Delta r_{z}\right\rangle=0$. We consider only an external force applied either parallel or antiparallel to the temperature gradient $\vec{\nabla} T$. Then the value of $Q^{* \prime}$ can be found from

$$
Q^{* \prime}=\left(\vec{F}_{e x t}^{0} \cdot \hat{k}\right) \frac{T}{\langle\vec{\nabla} T \cdot \hat{k}\rangle} .
$$

In Fig. 3, we show the computed values of $\left\langle\Delta r_{z}\right\rangle$ determined as a function of the magnitude of the external force $F_{\text {ext }}$. We include the result for the case $F_{\text {ext }}=0$ as described above. Each result is determined as a statistical average over 60 independent realizations. In the region of the system where the hydrogen atom is placed, the temperature gradient points in the negative-z direction. Although the statistical error in Fig. 3 is substantial, we also include a linear fit to the data and obtain the estimate $F_{\text {ext }}^{0}=0.005 \pm 0.002 \mathrm{eV} / \AA$. The value of $F_{e x t}^{0}$ is positive which indicates that the external force must be applied in the opposite direction as the gradient $\vec{\nabla} T$ in order to cancel the driving force for thermodiffusion. Finally, using 


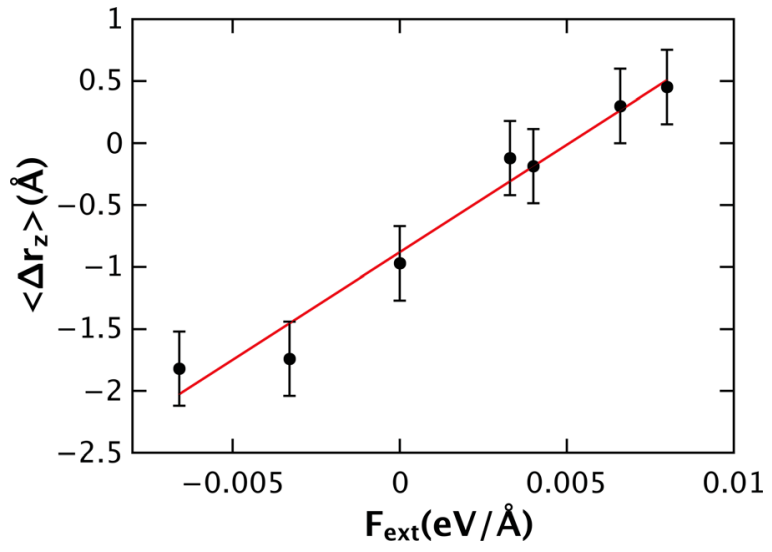

FIG. 3. Averaged displacements $\left\langle\Delta r_{z}\right\rangle$ as a function of the magnitude of the external force $F_{\text {ext }}$ after $20.47 \mathrm{ps}$ of simulation in a temperature gradient.

Eq. (28), the estimate $Q^{* \prime}=-0.31 \pm 0.10 \mathrm{eV}$. This result is quite consistent with the estimate $Q^{* \prime}=-0.33 \pm 0.10 \mathrm{eV}$ found only using the result for $\vec{F}_{\text {ext }}=0$.

In summary, direct simulation of thermodiffusion was observed by tracking the displacement of a hydrogen atom in the presence of a temperature gradient $\vec{\nabla} T$. The hydrogen atom clearly tends to drift in the direction of increasing temperature, corresponding to a negative value for the reduced heat of transport. The estimate $Q^{* \prime} \approx-0.3 \mathrm{eV}$ will be shown in Secs. IV B and IV C to be in reasonable agreement with predictions obtained using other methods. However, we believe that the approach used in this section, while adequate for establishing qualitative behavior and obtaining an estimate for $Q^{* \prime}$, may involve an important systematic error that, in the present case, results in over-prediction of the magnitude of $Q^{* \prime}$. In particular, in the direct method, the hydrogen atom can freely diffuse in the system and hence can explore a wide range of local temperatures. It is well known that the transport coefficients, in this case $L_{22}$ in particular, are temperature dependent. Hence, if the hydrogen atom happens to move into regions of higher temperature, its hopping rate will increase. By contrast, in the cases where the hydrogen atom moves into regions of lower temperature, its hopping rate will decrease. In the present case, we think that this could lead to larger displacements $\Delta r_{z}$ in the negative-z direction, thereby resulting in an overestimation of the magnitude of $Q^{* \prime}$. However, we have not found a way to test this hypothesis.

\section{B. Green-Kubo approach}

The theory of non-equilibrium thermodynamics demonstrates that the transport coefficients are directly related to time-correlation functions of the heat and mass fluxes. Generally known as the Green-Kubo formulae, ${ }^{3,4}$ application to thermodiffusion in solids is impractical in cases where the activation barriers are high in comparison to $k_{B} T$ and atom hops occur only very rarely. In the case of the $\mathrm{Pd}: \mathrm{H}$ system, Gillan ${ }^{6}$ successfully analyzed the Green-Kubo formulae and obtained a result for $Q^{* \prime}$, albeit with rather large statistical error. Here, we review the calculation and duplicate many of the results reported in Ref. 6 with improved statistical accuracy. We show that the value of $Q^{* \prime}$ depends on a consistent definition of the partial enthalpy $h_{2}$ of hydrogen. In Ref. 6, we believe an inconsistent definition for $h_{2}$ was chosen, resulting in $Q^{* \prime} \approx 0.04 \mathrm{eV}$ which is significant disagreement with the results reported here. We show that a correct analysis of $h_{2}$ brings the results of Ref. 6 for $Q^{* \prime}$ into very good agreement with our results.

We simulate a system with $N_{1}=108$ and $N_{2}=20$, which was also simulated in Ref. 6 . The mass flux $\vec{J}_{2}$ is given by Eq. (2), defined, as previously explained, in the center-ofmass reference frame. The approach in Ref. 6 was somewhat different. In particular, the results in Ref. 6 were determined using mass fluxes defined in a frame of reference fixed to the Pd lattice. In that case, the Pd mass flux is zero $\left(\vec{J}_{1}^{\prime}=0\right)$, and the hydrogen flux is given by

$$
\vec{J}_{2}^{\prime}=\sum_{i=1}^{N_{2}} \vec{v}_{i}-\frac{N_{2}}{N_{1}} \sum_{i=1}^{N_{1}} \vec{v}_{i}=\vec{J}_{2}-\frac{N_{2}}{N_{1}} \vec{J}_{1} .
$$

Then using Eq. (3) it is easily shown that

$$
\vec{J}_{2}^{\prime}=\vec{J}_{2}\left(1+\frac{N_{2} m_{2}}{N_{1} m_{1}}\right) \approx(1.0017) \vec{J}_{2},
$$

where the atomic velocities are defined with respect to the center-of-mass reference frame. We see that the mass flux $J_{2}^{\prime}$ used by Gillan in Ref. 6 is nearly equal to $\vec{J}_{2}$ as defined in the center-of-mass reference frame. Hence, we do not expect the results to depend in any significant fashion on whether the hydrogen flux is defined with respect to the center-ofmass or lattice-fixed reference frames.

The energy flux is computed in the center of mass reference frame by ${ }^{11}$

$$
\vec{J}_{q}=\sum_{i} \vec{v}_{i} \epsilon_{i}+\frac{1}{2} \sum_{i<j} \vec{r}_{i j}\left[\vec{F}_{i j} \cdot\left(\vec{v}_{i}+\vec{v}_{j}\right)\right]
$$

where the summations are over all particles, $\mathrm{Pd}$ and $\mathrm{H}$, and $\vec{F}_{i j}$ is the force on atom $i$ due to its interaction with atom $j$. The partial energy $\epsilon_{i}$ is given by

$$
\epsilon_{i}=\frac{1}{2} m_{i} \vec{v}_{i} \cdot \vec{v}_{i}+\frac{1}{2} \sum_{j \neq i} V_{i j}\left(r_{i j}\right),
$$

where $V_{i j}$ is the pair-potential interaction between particles $i$ and $j$ separated by a distance $r_{i j}$. The choice to divide the potential energy equally between the two interacting particles is arbitrary and should make no difference to theoretical predictions. Nevertheless, the choice above is quite standard and moreover was also used in Ref. 6. As discussed in Ref. 11, as long as the interactions are relatively shortranged, Eq. (32) is a suitable definition. The definition in Eq. (31) for the energy flux includes energy transport due to both heat conduction and mass transport. The heat flux, in contrast to the energy flux, describes only conduction. ${ }^{11}$ We use Eq. (12) to define the heat flux $\vec{J}_{q}^{\prime}$, which requires a sensible definition for the partial enthalpy of hydrogen $h_{2}$.

We next consider the calculation of the partial energies, $u_{1}$ and $u_{2}$, and partial enthalpies $h_{1}$ and $h_{2}$, for the $\mathrm{Pd}$ and 
hydrogen species, respectively. We take an approach that follows Ref. 11. For the partial energy of Pd, we use

$$
u_{1}=\frac{1}{N_{1}} \sum_{i=1}^{N_{1}}\left\langle\epsilon_{i}\right\rangle
$$

where $\epsilon_{i}$ is defined as in Eq. (32) and the summation includes only the $N_{1} \mathrm{Pd}$ atoms. The angle brackets indicate time averaging. Similarly, the partial energy of the hydrogen atoms is

$$
u_{2}=\frac{1}{N_{2}} \sum_{i=1}^{N_{2}}\left\langle\epsilon_{i}\right\rangle
$$

Then the total internal energy is given by

$$
U=N_{1} u_{1}+N_{2} u_{2} \text {. }
$$

The definitions of $h_{1}$ and $h_{2}$ should be consistent with $u_{1}$ and $u_{2}$. For Pd atoms, we define the partial enthalpy

$$
h_{1}=u_{1}+p_{1} \Omega \text {. }
$$

Similarly, the partial enthalpy of hydrogen is defined as

$$
h_{2}=u_{2}+p_{2} \Omega \text {. }
$$

The total enthalpy is then given as,

$$
H=N_{1} h_{1}+N_{2} h_{2}
$$

To determine the pressure terms $p_{1}$ and $p_{2}$, we first recall the definition for the elements of stress tensor $\pi_{\mu \nu}$ for a pair potential, ${ }^{11,12}$

$$
\pi_{\mu \nu} \Omega=\sum_{i} m_{i} v_{i, \mu} v_{i, \nu}+\frac{1}{2} \sum_{i} \sum_{j \neq i}\left(F_{i j, \mu} r_{i j, \nu}\right) .
$$

Here, the Greek indices $\mu$ and $\mu$ represent components in a Cartesian coordinate system. Hence, $v_{i, \mu}$ is a component of the velocity vector $\vec{v}_{i}$ for particle $i$. Similarly, $F_{i j, \mu}$ is a component of the vector $\vec{F}_{i j}$ which represents the force on particle $i$ due to its interaction with particle $j$, and $r_{i j, \mu}$ is a component of the vector $\vec{r}_{i j}=\vec{r}_{i}-\vec{r}_{j}$ which describes the relative position vectors of particles $i$ and $j$. The (time-averaged) total pressure $p$ is proportional the trace of the stress tensor

$$
p \Omega=\frac{1}{3} \sum_{\mu=1}^{3}\left\langle\pi_{\mu \mu}\right\rangle,
$$

which using Eq. (39) can also be written as

$$
p \Omega=\frac{1}{3}\left[\sum_{i} m_{i}\left\langle\vec{v}_{i} \cdot \vec{v}_{i}\right\rangle+\frac{1}{2} \sum_{i} \sum_{j \neq i}\left\langle\vec{F}_{i j} \cdot \vec{r}_{i j}\right\rangle\right] .
$$

In the definition of the quantities $p_{1}$ and $p_{2}$ used in the definitions of $h_{1}$ and $h_{2}$, we assume that the contributions due to the pairwise interactions are equally divided between the particles. Hence, we take

$$
\left\langle p_{i}\right\rangle \Omega=\frac{1}{3} m_{i}\left\langle\vec{v}_{i} \cdot \vec{v}_{i}\right\rangle+\frac{1}{6} \sum_{j \neq i}\left\langle\vec{F}_{i j} \cdot \vec{r}_{i j}\right\rangle .
$$

The partial pressure term $p_{1}$ due to $\mathrm{Pd}$ is then,

$$
p_{1}=\frac{1}{N_{1}} \sum_{i=1}^{N_{1}}\left\langle p_{i}\right\rangle
$$

and for hydrogen,

$$
p_{2}=\frac{1}{N_{2}} \sum_{i=1}^{N_{2}}\left\langle p_{i}\right\rangle
$$

Finally, the total pressure $p$ is related to the contributions $p_{1}$ and $p_{2}$ due to the $\mathrm{Pd}$ and hydrogen atoms

$$
p=N_{1} p_{1}+N_{2} p_{2} \text {. }
$$

The expressions for $\mathrm{H}$ and $\mathrm{U}$ and the individual contributions are related by

$$
\begin{aligned}
H & =N_{1} h_{1}+N_{2} h_{2}=N_{1}\left(u_{1}+p_{1} \Omega\right)+N_{2}\left(u_{2}+p_{2} \Omega\right) \\
& =U+p \Omega .
\end{aligned}
$$

Determined in this way, $h_{1}$ and $h_{2}$ are consistent with the definition of the energy terms $\epsilon_{i}$ defined by Eq. (32). In particular, it was shown in Ref. 11 that using the definition in Eq. (32) to determine the local energy density leads naturally to the local contribution to the internal pressure given by Eq. (42). In some studies, the partial enthalpy term is either neglected or incorrectly calculated, leading to errors in computed thermal conductivity values or other transport parameters. However, there are examples in the literature where the approach described above is used for computing partial enthalpy in binary liquids. ${ }^{13,14}$ Recently, a detailed study has been performed verifying that the approach above to compute the partial enthalpy terms results in good agreement between Green-Kubo and direct methods for computing thermal conductivity. ${ }^{15}$

We show in Table I the partial thermodynamic quantities computer for the $\mathrm{Pd}: \mathrm{H}$ system for $N_{1}=108 \mathrm{Pd}$ atoms and several different numbers of hydrogen atoms $N_{2}$. The temperature in each case corresponds to $T=980^{\circ} \mathrm{K}$, and the system volume is fixed independent of the number of hydrogen atoms $N_{2}$. For $N_{2}=20$, we find $h_{2}=2.038 \mathrm{eV}$. We find that $u_{2}$ is relatively independent of $N_{2}$, indicating that hydrogen-hydrogen interactions are fairly unimportant. For $u_{1}$, the value increases significantly as $N_{2}$ increases due to fact that half of the repulsive $\mathrm{Pd}-\mathrm{H}$ interactions are attributed to the $u_{1}$. Likewise, the energy $p_{1} \Omega$ depends fairly strongly on $N_{2}$, whereas $p_{2} \Omega$ depends only weakly on $N_{2}$.

In Ref. 6, a different approach was used to determine $h_{1}$ and $h_{2}$. In particular, Gillan determined $h_{2}$ using

$$
h_{2}=\left(\frac{\partial H}{\partial N_{2}}\right)_{p, T, N_{1}}=\left(\frac{\partial U}{\partial N_{2}}\right)_{\Omega, T, N_{1}}+v_{2} T\left(\frac{\partial p}{\partial T}\right)_{\Omega, N_{1}, N_{2}}
$$

with the partial volume of hydrogen in solution, 
TABLE I. Computed partial energies, enthalpies, and pressures compute for $N_{1}=108 \mathrm{Pd}$ atoms and different numbers $N_{2}$ of hydrogen atoms. Values were determined for a fixed volume $\Omega=27 a_{0}^{3}$ with $a_{0}=4.07 \AA$ independent of $N_{2}$, and a temperature $T=980^{\circ} \mathrm{K}$.

\begin{tabular}{lrcccccc}
\hline \hline$N_{1}$ & $N_{2}$ & $u_{1}$ & $u_{2}$ & $h_{1}$ & $h_{2}$ & $p_{1} \Omega$ & $p_{2} \Omega$ \\
\hline 108 & 0 & -0.563 & - & -0.314 & - & 0.249 & - \\
108 & 1 & -0.545 & 0.833 & -0.290 & 1.967 & 0.255 & 1.134 \\
108 & 10 & -0.464 & 0.832 & -0.103 & 1.999 & 0.361 & 1.167 \\
108 & 18 & -0.444 & 0.845 & -0.015 & 2.036 & 0.429 & 1.191 \\
108 & 19 & -0.410 & 0.836 & 0.045 & 2.022 & 0.455 & 1.186 \\
108 & 20 & -0.406 & 0.842 & 0.051 & 2.038 & 0.457 & 1.196 \\
108 & 21 & -0.410 & 0.846 & 0.054 & 2.044 & 0.464 & 1.198 \\
108 & 22 & -0.385 & 0.854 & 0.098 & 2.059 & 0.483 & 1.205 \\
\hline \hline
\end{tabular}

$$
v_{2}=\left(\frac{\partial \Omega}{\partial N_{2}}\right)_{p, T, N_{1}}
$$

The main calculation required to use these expressions is the total energy $U$ as a function of the $N_{2}$. This approach yields significantly different values than the values reported in Table I. In particular, applying Eq. (47) to the potentials used in this paper, we obtain $h_{2}=1.733 \mathrm{eV}$ in contrast to the value $h_{2}=2.038 \mathrm{eV}$ in Table I. In Ref. $6, h_{2}=2.02 \mathrm{eV}$ was obtained. While the approach in Ref. 6 for determining partial enthalpy is not incorrect, we believe that it is incorrect in this context. In particular, the definition for $h_{2}$ used by Gil$\operatorname{lan}^{6}$ is not consistent with the expressions in Ref. 11, which were derived assuming the same definition of the local energy term $\epsilon_{i}$ given by Eq. (32). Below, we will see that the results reported here are, in fact, in agreement with Ref. 6, when the correct value for $h_{2}$ is used.

The transport parameters are determined by integration of the time-correlation functions,

$$
\begin{aligned}
& \zeta_{22}(\tau)=\frac{1}{3 \Omega} \int_{0}^{\tau}\left\langle\vec{J}_{2}(\tau) \cdot \vec{J}_{2}(0)\right\rangle d \tau, \\
& \zeta_{2 q}(\tau)=\frac{1}{3 \Omega} \int_{0}^{\tau}\left\langle\vec{J}_{2}(\tau) \cdot \vec{J}_{q}(0)\right\rangle d \tau, \\
& \zeta_{q 2}(\tau)=\frac{1}{3 \Omega} \int_{0}^{\tau}\left\langle\vec{J}_{q}(\tau) \cdot \vec{J}_{2}(0)\right\rangle d \tau, \\
& \zeta_{q q}(\tau)=\frac{1}{3 \Omega} \int_{0}^{\tau}\left\langle\vec{J}_{q}(\tau) \cdot \vec{J}_{q}(0)\right\rangle d \tau .
\end{aligned}
$$

In particular,

$$
\begin{gathered}
L_{22}=\lim _{\tau \rightarrow \infty} \zeta_{22}(\tau), \\
L_{2 q}=L_{q 2}=\lim _{\tau \rightarrow \infty} \zeta_{2 q}(\tau)=\lim _{\tau \rightarrow \infty} \zeta_{q 2}(\tau), \\
L_{q q}=\lim _{\tau \rightarrow \infty} \zeta_{q q}(\tau) .
\end{gathered}
$$

Then the value of the heat of transport $Q^{*}$ is found from

$$
Q^{*}=\frac{L_{2 q}}{L_{22}} .
$$

If instead the definition $\vec{J}_{q}^{\prime}$ for the heat flux given in Eq. (12) is used, the relevant correlation functions are

$$
\begin{aligned}
& \zeta_{2 q}^{\prime}(\tau)=\frac{1}{3 \Omega} \int_{0}^{\tau}\left\langle\vec{J}_{2}(\tau) \cdot \vec{J}_{q}^{\prime}(0)\right\rangle d \tau, \\
& \zeta_{q 2}^{\prime}(\tau)=\frac{1}{3 \Omega} \int_{0}^{\tau}\left\langle\vec{J}_{q}^{\prime}(\tau) \cdot \vec{J}_{2}(0)\right\rangle d \tau, \\
& \zeta_{q q}^{\prime}(\tau)=\frac{1}{3 \Omega} \int_{0}^{\tau}\left\langle\vec{J}_{q}^{\prime}(\tau) \cdot \vec{J}_{q}^{\prime}(0)\right\rangle d \tau,
\end{aligned}
$$

while $\zeta_{22}(\tau)$ is unchanged. The correlation functions are related by

$$
\begin{gathered}
\zeta_{2 q}^{\prime}(\tau)=\zeta_{2 q}(\tau)-h_{2} \zeta_{22}(\tau), \\
\zeta_{q q}^{\prime}(\tau)=\zeta_{q q}(\tau)-h_{2}\left(\zeta_{2 q}(\tau)+\zeta_{q 2}(\tau)\right)+h_{2}^{2} \zeta_{22}(\tau) .
\end{gathered}
$$

From these expressions, the relationship between the transport coefficients given in Eqs. (16) and (17) can be obtained. Then, the reduced heat of transport $Q^{* \prime}$ is found from

$$
Q^{* \prime}=\frac{L_{2 q}^{\prime}}{L_{22}},
$$

where $L_{2 q}^{\prime}=\lim _{\tau \rightarrow \infty} \zeta_{2 q}^{\prime}(\tau)$. As we have seen previously, $Q^{* \prime}$ and $Q^{*}$ are differ by the partial enthalpy $h_{2}$, and $Q^{* \prime}=Q^{*}-h_{2}$. Thus, $Q^{*}$ can be easily determined independent of the definition of $h_{2}$, whereas $Q^{* \prime}$ depends on a correct value for $h_{2}$.

To determine the transport coefficients, we performed a total of 360 independent equilibrium MD simulations at an average temperature $T=980^{\circ} \mathrm{K}$. In each simulation, $10^{4}$ MD steps were used to equilibrate the system, followed by $2 \times 10^{5}$ steps to determine the time correlation functions. The total amount of simulation time used for averaging was $29.8 \mathrm{~ns}$. In Ref. 6, the total simulation time used for averaging was $2.0 \mathrm{~ns}$ over 16 independent simulations. The statistical errors reported in Ref. 6 for the calculation of $Q^{* \prime}$ where comparable to the computed value. Owing to the much longer simulation time reported here, we estimate that the statistical error should be reduced by about a factor of 3-4.

We show in Table II the computed transport coefficients for a different integration times $\tau$ along with an estimate of the numerical error. The error was estimated by determining the standard deviation for 4 independent segments of the entire data set. Each of the transport parameters appears fairly well converged after $\tau=2.0 \mathrm{ps}$ of integration time with acceptable error. At $\tau=1.2 \mathrm{ps}$, the transport parameters also appear well converged with the exceptions of $L_{q q}$ and $L_{q q}^{\prime}$. For longer integration times $\tau$, the estimated error becomes comparable to the parameters themselves. Consequently, there is no clear evidence for correlations beyond $\tau=2.0 \mathrm{ps}$. Hence, in the following, we refer in particular to the values computed for $\tau=2.0 \mathrm{ps}$. However, we show data in the figures for integration times extending until $\tau=20 \mathrm{ps}$.

In Fig. 4, we show the integrated correlation function $\zeta_{22}(\tau)$ as a function of integration time $\tau$. The results are in 
TABLE II. Computed transport parameters obtained using the Gillain potential ${ }^{5}$ with the smoothing described in the text. Results are given for different values of the integration time $\tau$.

\begin{tabular}{llllll}
\hline \hline$\tau(\mathrm{ps})$ & $L_{22}\left(\mathrm{ps}^{-1} \AA^{-1}\right)$ & $L_{2 q}\left(\mathrm{eV} \mathrm{ps}^{-1} \AA^{-1}\right)$ & $L_{2 q}^{\prime}\left(\mathrm{eV} \mathrm{ps}^{-1} \AA^{-1}\right)$ & $L_{q q}\left(\mathrm{eV}^{2} \mathrm{ps}^{-1} \AA^{-1}\right)$ & $L_{q q}^{\prime}\left(\mathrm{eV}^{2} \mathrm{ps}^{-1} \AA^{-1}\right)$ \\
\hline 1.2 & $0.033 \pm 0.001$ & $0.060 \pm 0.001$ & $-0.007 \pm 0.001$ & $0.175 \pm 0.003$ & $0.066 \pm 0.005$ \\
2.0 & $0.034 \pm 0.003$ & $0.062 \pm 0.001$ & $-0.007 \pm 0.001$ & $0.186 \pm 0.015$ & $0.073 \pm 0.007$ \\
5.0 & $0.038 \pm 0.005$ & $0.069 \pm 0.008$ & $-0.009 \pm 0.008$ & $0.213 \pm 0.033$ & $0.091 \pm 0.022$ \\
10.0 & $0.036 \pm 0.006$ & $0.064 \pm 0.017$ & $-0.009 \pm 0.017$ & $0.198 \pm 0.033$ & $0.086 \pm 0.038$ \\
\hline
\end{tabular}

reasonable agreement with those reported in Ref. 6 for times below the $1.2 \mathrm{ps}$. In particular, at $\tau=1.2 \mathrm{ps,} \mathrm{Gillan}$ reported a value $L_{22}=0.029 \mathrm{ps}^{-1} \AA^{-1}$ compared to the result here $L_{22}=0.033 \mathrm{ps}^{-1} \AA^{-1}$. For the value $L_{22}=0.034 \mathrm{ps}^{-1} \AA^{-1}$ obtained for $\tau=2.0 \mathrm{ps}$ of integration, we obtain the diffusion coefficient $D=3.09 \pm 0.31 \mathrm{~cm}^{2} \mathrm{~s}^{-1}$, in good agreement with the value $D=3.03 \mathrm{~cm}^{2} \mathrm{~s}^{-1}$ obtained using the Einstein relation described in Sec. III. Results for times beyond $1.2 \mathrm{ps}$ were not reported in Ref. 6 . We find that $\zeta_{22}(\tau)$ increases gradually well beyond $1.2 \mathrm{ps}$. This could be due to correlated diffusion of the $\mathrm{N}_{2}=20$ hydrogen atoms. For example, the hopping of a hydrogen atom may either act to block or open a site, initiating or inhibiting diffusion of another hydrogen. However, the long-time correlations are not larger than the statistical error, and we conclude that longer simulation time is required to adequately resolve this question.

In Fig. 5, the integrated cross-correlation function $\zeta_{2 q}(\tau)$ is shown. It appears that $\zeta_{2 q}(\tau)$ mostly converges within less than 1 ps of integration time. The correlation between the energy flux and the hydrogen flux is quite strong, demonstrating the important role played by hydrogen in energy transport. For $\tau=2.0 \mathrm{ps}$ of integration time, we obtain a value $L_{2 q}=0.062 \pm 0.001 \mathrm{eVps}^{-1} \AA^{-1}$. A value for this quantity was not reported in Ref. 6. Using $L_{2 q}$ and $L_{22}$ determined from $\tau=2.0 \mathrm{ps}$ of integration time, we obtain the prediction $Q^{*}=1.83 \pm 0.04 \mathrm{eV}$.

The function $\zeta_{2 q}^{\prime}(\tau)$ is shown in Fig. 6. In contrast to $\zeta_{2 q}(\tau)$ shown in Fig. 5, the values are small and consistently negative. We use the value $h_{2}=2.038 \mathrm{eV}$ reported in Table I. The very small values suggest that correlation effects

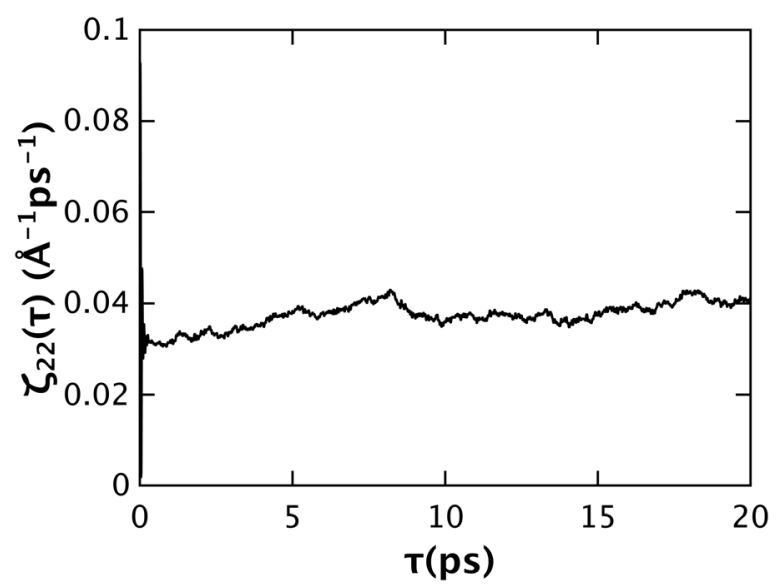

FIG. 4. The integrated correlation function $\zeta_{22}(\tau)$ as a function of integration time $\tau$. between the heat flux and mass transport are in fact quite small. The results differ here from what was reported in Ref. 6. In particular, in Ref. 6, it was found that $\zeta_{2 q}^{\prime}(\tau)$ was positive for times to $\tau=1.2 \mathrm{ps}$, resulting in the prediction $L_{2 q}^{\prime}$ $=0.001 \mathrm{eVps}^{-1} \AA^{-1}$. By contrast, we obtain $L_{2 q}^{\prime}=-0.007$ $\pm 0.001 \mathrm{eVps}^{-1} \AA^{-1}$ for $\tau=1.2 \mathrm{ps}$. The value $L_{2 q}^{\prime}$ we obtain is unchanged for $\tau=2.0 \mathrm{ps}$ of integration time. Using the results for $\tau=2.0 \mathrm{ps}$ of integration time, we obtain for the reduced heat of transport $Q^{* \prime}=-0.21 \pm 0.04 \mathrm{eV}$.

The positive value for $L_{2 q}^{\prime}$ found in Ref. 6 resulted in the prediction $Q^{* \prime} \approx 0.04 \mathrm{eV}$. We believe that the apparent disagreement is due to the inappropriate definition of $h_{2}$ used by Gillan. ${ }^{6}$ We first note that, given the value $h_{2}=2.02 \mathrm{eV}$ reported by Gillan, ${ }^{6}$ that the heat of transport $Q^{*}=Q^{* \prime}$ $+h_{2}=2.06 \mathrm{eV}$. This is to be compared to $Q^{*}=1.83 \mathrm{eV}$ determined above. This comparison is completely independent of $h_{2}$.

We can also obtain a correct value for $Q^{* \prime}$ from the data reported in Ref. 6 and a correct calculation of $h_{2}$. Using the potentials defined in Ref. 5 without smoothing, we use the correct definition for $h_{2}$ given above by Eqs. (34), (37), (42), and (44) and obtain the value $h_{2}=2.29 \mathrm{eV}$. Using this value, the results in Ref. 6 are found to correspond to a reduced heat of transport $Q^{* \prime}=-0.23 \pm 0.02 \mathrm{eV}$. This is extremely close to the result reported here, suggesting that our results are actually in very good agreement with those reported by Gillan in Ref. 6, and that the differences are almost entirely due to the incorrect value of $h_{2}$. The primary affect of the smoothing the potentials seems to be to introduce a downward shift of about $0.23-0.25 \mathrm{eV}$ in the values of $h_{2}$ and $Q^{*}$. This is comparable, if not exactly equal, to the downward

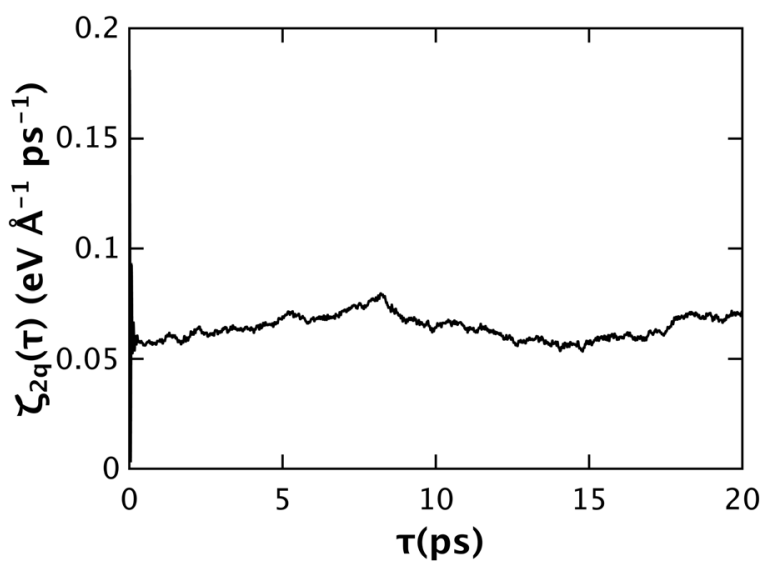

FIG. 5. The integrated correlation function $\zeta_{2 q}(\tau)$ as a function of integration time $\tau$. 


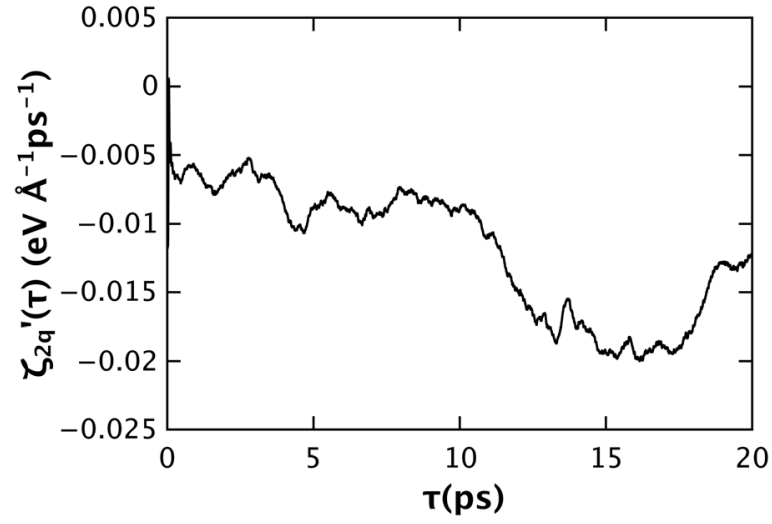

FIG. 6. The integrated correlation function $\zeta_{2 q}^{\prime}(\tau)$ as a function of integration time $\tau$.

shift of $0.29 \mathrm{eV}$ in the energy of a hydrogen atom occupying an interstitial site at $T=0 \mathrm{~K}$. In the calculation of the reduced heat of transport $Q^{* \prime}=Q^{*}-h_{2}$, the downward shift in both quantities appears to cancel.

Finally, the integrated functions $\zeta_{q q}(\tau)$ and $\zeta_{q q}^{\prime}(\tau)$ are shown in Fig. 7. As with the other integrated correlation functions, the values converge fairly quickly. The results demonstrate that the energy flux associated with hydrogen diffusion is comparable to conductive heat transfer. The results in Fig. 7 can be used to estimate the thermal conductivity $\kappa$. In particular, we use the results for $\zeta_{q q}^{\prime}(\tau)$ to determine the thermal conductivity from

$$
\kappa=\frac{L_{q q}^{\prime}}{k_{B} T^{2}} .
$$

Using the value of $\zeta_{q q}^{\prime}=0.073 \mathrm{eV}^{2} \mathrm{ps}^{-1} \AA^{-1}$ after an integration time $\tau=2.0 \mathrm{ps}$ for the transport coefficient $L_{q q}^{\prime}$, the estimate $\kappa=1.36 \pm 0.14 \mathrm{Wm}^{-1^{\circ}} \mathrm{K}^{-1}$ is obtained. In Ref. 6 , the value $\kappa=1.1 \mathrm{Wm}^{-1}{ }^{\circ} \mathrm{K}^{-1}$ was reported. The differences here are due in part to the incorrect definition of $h_{2}$. However, we also found that the integrated correlation function did not converge to its asymptotic value within the $\tau=1.2 \mathrm{ps}$ reported in Ref. 6 . The value of $\kappa$ can also be obtained from the value of the applied heat flux and the time-averaged tem-

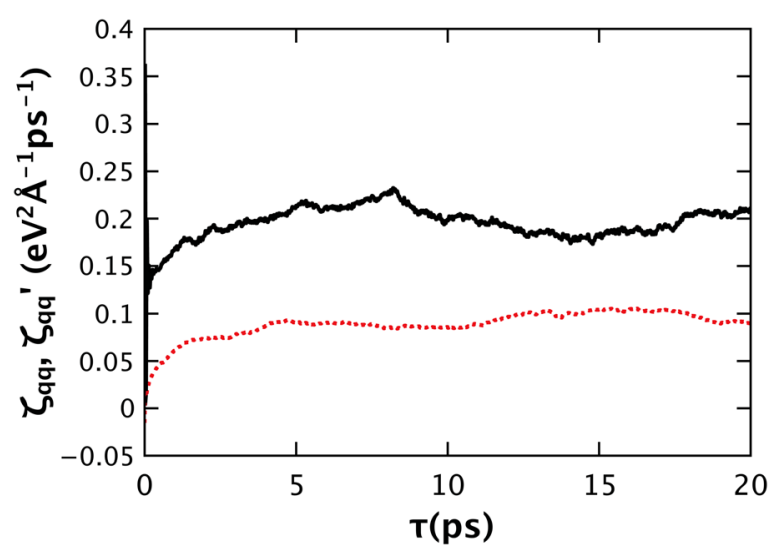

FIG. 7. The integrated correlation functions $\zeta_{q q}(\tau)$ (solid black line) and $\zeta_{q q}^{\prime}(\tau)$ (dotted red line) as a function of integration time $\tau$. perature gradients shown in Fig. 1 and discussed in Sec. IV A. It is well known that this approach yields results that exhibit very strong finite-size effects. ${ }^{16-18}$ Nevertheless, using Fourier's law we obtain the estimate $\kappa=$ $1.4 \mathrm{Wm}^{-1^{\circ}} \mathrm{K}^{-1}$ which is in agreement with the Green-Kubo result. Finite-size effects tend to be more significant when the phonon mean free path is long and $\kappa$ is relatively high, which does not correspond to the $\mathrm{Pd}: \mathrm{H}$ system simulated here. Finally, it is not expected that these values for $\kappa$ for lattice heat conduction will be in good agreement with experimental measurements of $\kappa$, since in metals electronic heat conduction is known to be dominant.

\section{Constrained-dynamics approach}

In Ref. 8, an approach was developed that uses a constraint force $\vec{F}_{c}$ to move an atom through the transition state. The approach was applied in Ref. 8 to vacancy thermodiffusion in a Lennard-Jones crystal. The basic idea is to compute the net work $W_{1 \rightarrow 2}$ required to displace an atom from $z_{1}$ to $z_{2}$, the initial and final local-equilibrium positions, through the transition state in the presence of a temperature gradient. It was shown in Ref. 8 that the computed work can be used to determine $Q^{* \prime}$ from

$$
Q^{* \prime}=W_{1 \rightarrow 2} \frac{T}{\Delta T}
$$

where $\Delta T$ is the difference in the local temperature between points $z_{1}$ and $z_{2}$. Here, we apply this approach to the $\mathrm{Pd}: \mathrm{H}$ system. Before presenting results, we first address some questions about the methodology and attempt to give further justification than was given in Ref. 8 .

The results determined using the direct approach described in Sec. IV A show that thermodiffusion in the $\mathrm{Pd}: \mathrm{H}$ system occurs as a result of an average force felt by the hydrogen atom due to the temperature gradient. It was furthermore shown how this force could be determined and then related to $Q^{* \prime}$. Moreover, it is possible to determine an external force $\vec{F}_{\text {ext }}^{0}$ that on average cancels the driving force due to an applied temperature gradient. We begin with Eq. (28) which defines $\vec{F}_{\text {ext }}^{0}$. We integrate this equation along a path between two points $z_{1}$ and $z_{2}$

$$
\int_{z_{1}}^{z_{2}} \vec{F}_{e x t}^{0} \cdot \hat{k} d z=Q^{* \prime} \int_{z_{1}}^{z_{2}}\left(\frac{\vec{\nabla} T}{T}\right) \cdot \hat{k} d z .
$$

The left-hand side is just the work done by the external force as the particle is displaced from $z_{1}$ to $z_{2}$ which we denote as $W_{1 \rightarrow 2}$. Because $\vec{F}_{\text {ext }}^{0}$ is uniform, $W_{1 \rightarrow 2}=F_{e x t}^{0}\left(z_{2}-z_{1}\right)$. The right-hand side, to first order, is given by

$$
Q^{* \prime} \int_{z_{1}}^{z_{2}}\left(\frac{\vec{\nabla} T}{T}\right) \cdot \hat{k} d z \approx Q^{* \prime} \frac{\Delta T}{T} .
$$

Hence, we obtain Eq. (64). In the derivation given here for Eq. (64), there is no argument that requires the external force to be uniform. We propose here that Eq. (64) also applies to 
the work done by the non-uniform constraint force $\vec{F}_{c}$. Both $\vec{F}_{c}$ and $\vec{F}_{\text {ext }}^{0}$ represent a conservative external force. The constraint force $\vec{F}_{c}$ exactly cancels the total interatomic force felt by the hydrogen atom along the direction of constraint (in this case along a [110] direction), and hence exactly cancels the driving force due the temperature gradient at each point between $z_{1}$ and $z_{2}$. By contrast, $\vec{F}_{\text {ext }}^{0}$ only acts to cancel the thermodiffusion driving force on average. The relationship between the constraint force $\vec{F}_{c}$ and the external force $\vec{F}_{e x t}^{0}$ is found by equating the work $W_{1 \rightarrow 2}$ in either case

$$
W_{1 \rightarrow 2}=\int_{z_{1}}^{z_{2}}\left\langle\vec{F}_{c}(z) \cdot \hat{k}\right\rangle d z=F_{e x t}^{0}\left(z_{2}-z_{1}\right) .
$$

Hence, using the approach in Ref. 8 to compute $W_{1 \rightarrow 2}$ from the z-component of the time-averaged constraint force $\left\langle\vec{F}_{c} \cdot \hat{k}\right\rangle$, direct comparison can be made to the estimated $F_{\text {ext }}^{0}$ determined in Sec. IV A.

In the constrained-dynamics simulations, the system size and temperature gradient are exactly the same as in the direct thermodiffusion simulations in Sec. IV A. At the beginning of each MD simulation, the hydrogen atom is displaced by the desired amount from the equilibrium position $z_{1}$. In addition, its initial velocity component along the $\mathrm{z}$ direction, corresponding to the [110]-direction in the fcc lattice, is set to zero. During the MD simulation, the force $\vec{F}$ on the hydrogen atom due to the neighboring Pd atoms is computed. The component of $\vec{F}$ along the z-direction is determined from

$$
F_{z}=\vec{F} \cdot \hat{k}
$$

The constraint force is added so that the motion of the hydrogen atom is constrained to a (110) plane. In particular, the constraint force is

$$
\vec{F}_{c}=-F_{z} \hat{k}
$$

The hydrogen follows dynamics with a force $\vec{F}^{\prime}$ given by

$$
\vec{F}^{\prime}=\vec{F}+\vec{F}_{c} .
$$

Because $\vec{F}^{\prime}$ has no component along the [110] direction, there is no motion in that direction. However, the hydrogen atom is not constrained in directions perpendicular to [110]. In this way, the hydrogen atom samples many possible transition paths, while it preferentially explores the saddle point of the transition. Finally, so that the center of mass of the system does not move with the application of $\vec{F}_{c}$ to the hydrogen atom, an equal but opposite force is applied to the Pd atoms. We choose to apply the same force to each of the Pd atoms, so that the force on Pd atom $i$ is given by,

$$
\vec{F}_{i}^{\prime}=\vec{F}_{i}-\frac{\vec{F}_{c}}{N_{1}} .
$$

At each point along the path, the system is first equilibrated at $T=980^{\circ} \mathrm{K}$ for $10^{5} \mathrm{MD}$ steps corresponding to about $41.4 \mathrm{ps}$. Afterwards, the temperature gradient is imposed and the system is run for $2 \times 10^{6} \mathrm{MD}$ steps or $0.829 \mathrm{~ns}$. After an

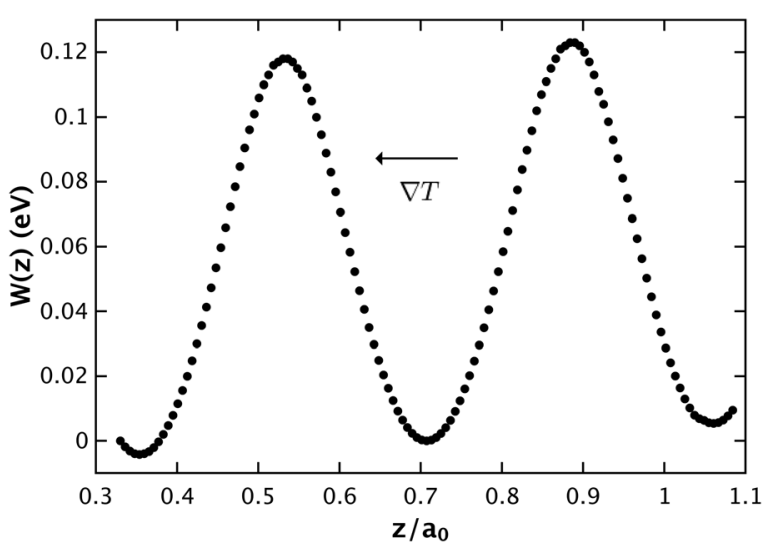

FIG. 8. The integrated work $W(z)$ required to drive a single hydrogen atom in an temperature gradient as a function of position $z$ in units of the lattice parameter $a_{0}=4.07 \AA$. The direction of the temperature gradient $\vec{\nabla} T$ is shown by an arrow.

initial time of $8.28 \mathrm{ps}$ to establish the temperature gradient in steady state, the constraint force is averaged over the remainder of the simulation time.

In Fig. 8, we show the integrated work $W(z)$ required to displace a single hydrogen atom in the presence of a temperature gradient. The work $W(z)$ was computed for 129 different displacements $z$ of the hydrogen atom. Figure 8 clearly shows a trend that $W(z)$ increases overall as the hydrogen atom is displaced in the opposite direction of the temperature gradient. Along the path of length $a_{0} / \sqrt{2}$ corresponding to the distance between two octahedral interstitial sites, we find that the hydrogen actually finds another energy minimum. The overall path of the hydrogen atom is depicted in Fig. 9. However, the path shown in Fig. 9 is simplified from the actual trajectory, since the hydrogen atom can move freely perpendicular to the [110] direction. In fact, in the course of a simulation, we find that the hydrogen atom freely diffuses perpendicular to the constraint direction.

In the initial equilibration period at $T=980^{\circ} \mathrm{K}$ before the temperature gradient is imposed, we compute the work $W_{\vec{\nabla} T=0}(z)$ done by the constraint force. In Fig. 10, we subtract from $W(z)$ shown in Fig. 8 the work $W_{\vec{\nabla} T=0}(z)$. This difference clearly demonstrates the thermodiffusion driving force. In particular, the general increasing trend with increasing $z$ is consistent with the existence of a thermodiffusion driving force tending to push the hydrogen towards higher temperatures (i.e., with the temperature gradient $\vec{\nabla} T$ ). From the difference between $W\left(z=z_{1}\right)$ at the minima near $z_{1}=$ $0.35 a_{0}$ and $W\left(z=z_{2}\right)$ at the minima near $z_{2}=1.06 a_{0}$, we obtain the estimate $W_{1 \rightarrow 2}=9.6 \times 10^{-3} \mathrm{eV}$. To determine the temperature difference $\Delta T$, we use the $\vec{\nabla} T \cdot \hat{k}=15.8^{\circ} \mathrm{K}^{-1}$ obtained in Sec. IV A for the approximate magnitude of the temperature gradient and find $\Delta T \approx 45.5^{\circ} \mathrm{K}$. From Eq. (64), we hence obtain $Q^{* \prime}=-0.21 \pm 0.02 \mathrm{eV}$. The error is estimated from uncertainty in the local temperature and the temperature gradient. However, there is a need for a detailed study of this method including to elucidate possible nonlinear and finite-size effects, and the error estimate here is quite tentative. This is discussed further in Sec. V. Nevertheless, this result is in agreement with the Green-Kubo result 


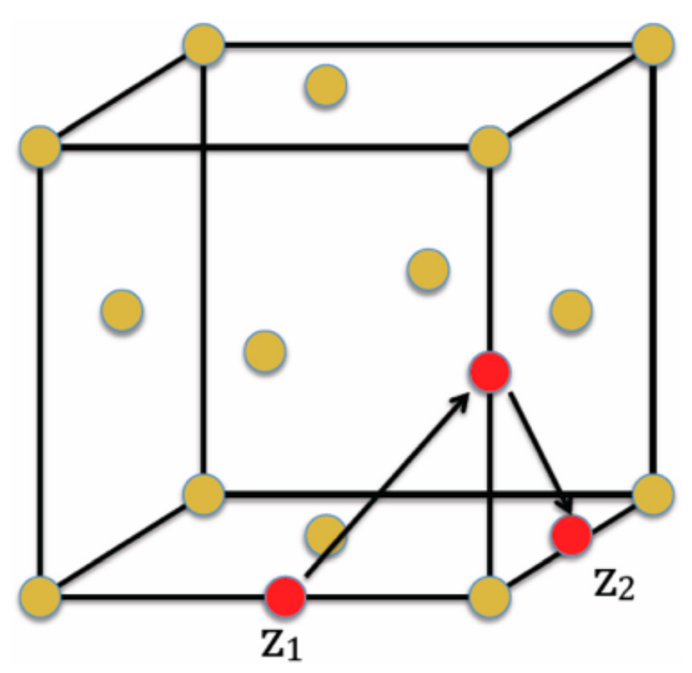

FIG. 9. Path taken by the hydrogen atom for the constrained-dynamics results shown in Fig. 8. The hydrogen atom essentially explores three local minima along the path from $z_{1}$ to $z_{2}$ The arrows do not represent a realistic trajectory, since the hydrogen atom actually freely diffuses perpendicular to the $[110]$ constraint direction.

$Q^{* \prime}=-0.21 \pm 0.04 \mathrm{eV}$, although there is a noticeable quantitative difference from the prediction $Q^{* \prime} \approx-0.30 \mathrm{eV}$ using the results of direct thermodiffusion simulations.

The work $W(z)$ shown in Fig. 8 is very closely related to the free energy profile. In equilibrium conditions $\vec{\nabla} T=0$, it is in fact equal to the free energy $A(z)$ corresponding to the hydrogen atom at position $z$. Therefore, the barriers in Fig. 8 are very close to the activation free energy $\Delta A_{a c t}$. We obtain the value $\Delta A_{a c t}=0.12 \mathrm{eV}$, which is significantly lower than $\Delta U_{a c t}=0.25 \mathrm{eV}$ found in Sec. IV A. This difference indications strong entropic effects along the diffusion path. In fact, using the expression for the entropy of activation

$$
\Delta S_{a c t}=\frac{\Delta U_{a c t}-\Delta A_{a c t}}{T},
$$

we obtain the estimate $\Delta S_{a c t}=1.54 k_{B}$. We believe that the detailed energetics and entropic effects along the diffusion

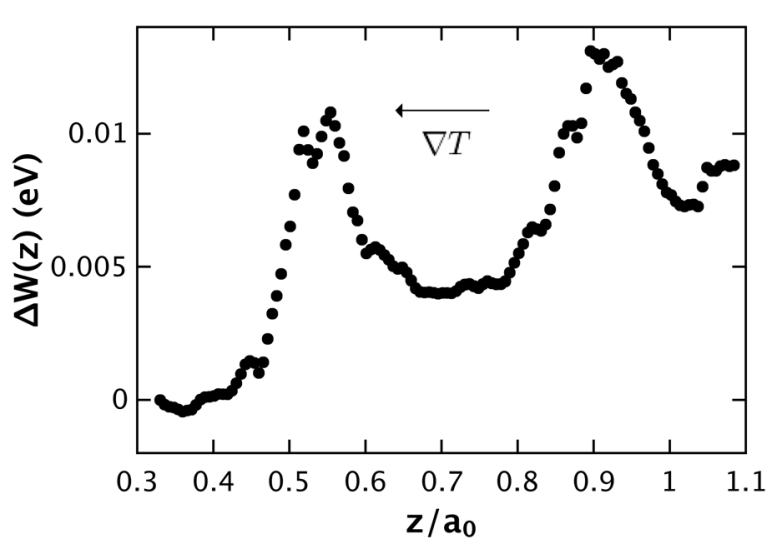

FIG. 10. The difference between the work $W(z)$ shown in Fig. 8 and the work $W_{\vec{\nabla} T=0}(z)$ computed with no temperature gradient as a function of position $z$ in units of the lattice parameter $a_{0}=4.07 \AA$. The direction of the temperature gradient $\vec{\nabla} T$ is shown by an arrow. The figure is consistent with a force tending to drive the hydrogen atom in the negative $z$-direction (i.e. with the temperature gradient in the direction of increasing temperature). pathway should be directly connected to the thermodiffusion behavior. Becomes this information emerges naturally from constrained-dynamics simulations, we believe this is another reason to be hopeful that new insight can be obtained.

Finally, we examine the predicted relationship given by Eq. (67) between the average constraint force and the external force $F_{e x t}^{0}$. Using the value $W_{1 \rightarrow 2}=9.6 \times 10^{-3} \mathrm{eV}$ determined above for the constraint force, we obtain the prediction $F_{\text {ext }}^{0}=0.003 \mathrm{eV} / \AA$. This is to be compared to the value $F_{\text {ext }}^{0}=0.005 \pm 0.002 \mathrm{eV} / \AA$ obtained in Sec. IV A. The agreement appears to be only approximate, which was expected considering the disagreement between $Q^{* \prime}$ determined using the direct method described in Sec. IV A and the constrained-dynamics results.

\section{SUMMARY AND CONCLUSIONS}

Different computational methodologies were explored for computing the heat of transport of hydrogen in a Pd lattice. Using direct simulation of thermodiffusion, the GreenKubo formulae, and the constrained-dynamics approach, the reduced heat of transport $Q^{* \prime}$ was found to be consistently negative in the range between $-0.20 \mathrm{eV}$ to $-0.30 \mathrm{eV}$. In the case of direct simulation of thermodiffusion, the results unambiguously demonstrate that hydrogen is driven in the direction of increasing local temperature. This provide important validation for the Green-Kubo and constraineddynamics results. The consistency of the sign and magnitude of $Q^{* \prime}$ suggests that each of these methods provide reasonable results. Disagreement with previously published results by Gillan ${ }^{6}$ was shown to be almost entirely due to an incorrect definition of $h_{2}$.

For the $\mathrm{Pd}: \mathrm{H}$ system, it is not clear which approach gives an advantage in accuracy or computational efficiency. The direct simulations of thermodiffusion result in $Q^{* \prime} \approx-0.30 \mathrm{eV}$, which is significantly larger magnitude than the prediction from Green-Kubo and constraineddynamics simulation $Q^{* \prime}=-0.21 \mathrm{eV}$. As discussed previously, we think that the differences here might be due to the fact that in the direct simulations, the hydrogen atom is free to explore a wide range of local temperatures and hence strongly varying local mobility. However, this hypothesis has not been directly confirmed.

In the case of the constrained-dynamics approach, there is a need to more thoroughly explore the practical aspects of the methodology. For example, it may be that there are nonlinear and finite-size effects that introduce error. We plan a study in the near future where variations in system size and the magnitude of the applied heat flux are explored. It should then be possible to determine the relative merits of the Green-Kubo and constrained-dynamics approaches. At present, it is clear that the constrained-dynamics method requires simulations of a larger system than in the Green-Kubo method. However, it should be noted that $\mathrm{Pd}: \mathrm{H}$ represents a special case due to the fast diffusion of the hydrogen. In other problems, for example, the thermodiffusion of vacancies or vacancy-mediated thermodiffusion in a binary metal alloy, it is clear that, of the three approaches studied here, only the constrained-dynamics approach first developed in 
Ref. 8 is capable of providing results. Another advantage of the constrained-dynamics approach is that basic information about the thermodynamics of the transition path emerges, including the activation free energy and entropy, which may in some way be correlated to $Q^{* \prime}$.

Another approach developed first by Gillan ${ }^{19-21}$ for $T=0^{\circ} \mathrm{K}$ and later modified for finite temperatures ${ }^{22}$ has not been considered in this article. In this approach, the diffusing particle is constrained at the saddle point and then is later released. As the particle moves towards an equilibrium point, the heat current is computed and then related to $Q^{* \prime}$. This approach has been applied in several studies to the study the thermodiffusion of vacancies in fcc crystals. ${ }^{23-25}$ As with the constrained-dynamics method, the Gillan approach at finite temperature has the advantage of being applicable to systems where diffusion is too slow to be observed on a typical MD time scale. However, we are aware of no studies that have been done to validate the finite-temperature method in Ref. 22 by comparison to other methods. It would be interesting to compare the results obtained using that approach to the Pd:H system where we now have quite thorough and accurate results.

The results here are in strong disagreement with experimental values, indicating that the empirical potential for the $\mathrm{Pd}: \mathrm{H}$ system is inadequate in some critical respect. For the experimental measurement in the temperature range $670-830^{\circ} \mathrm{K}$, the value $0.065 \mathrm{eV}$ was reported in Ref. 26 . This suggests that hydrogen tends to diffuse form the hot end to the cold end. In this article, the prime objective was to provide insight and to validate different computational methodologies including in particular the constrained-dynamics method. However, it is still an important goal in the future to generate computational results that can make accurate, materials-specific predictions. However, because insight into thermodiffusion remains incomplete, we cannot say what are the key quantities that a model needs to accurately reproduce in order to predict values of $Q^{* \prime}$ that are in agreement with experiment. In the $\mathrm{Pd}: \mathrm{H}$ system, another important aspect might be at play. In particular, heat conduction in metals is dominated by electrons, and it is not known whether an electron wind or thermoelectric driving force might act on the hydrogen atoms. Because hydrogen atoms have a very small mass, this effect might be relevant. By contrast, this contribution is likely to be small for vacancy-mediated thermodiffusion in a binary alloy.

However, there may be some insight into the Pd:H system which explains the observation of hydrogen flow from high to low temperatures. In particular, Asaro and coworkers put forth simple arguments to describe the thermodiffusion of interstitials, including in fcc solids. ${ }^{27}$ The arguments basically relate to the uptake and release of heat during as an interstitial moves from the initial to the final state. Their assumption is that, as the $\mathrm{H}$ moves from the transition state to the final equilibrium state, it releases an energy approximately equal to the migration energy. Assuming that the energy is radiated symmetrically, this would lead to a positive value for the heat of transport. This qualitative prediction is apparently in agreement with experiment but does not explain the simulation results here, where $Q^{* \prime}$ is negative.
One possible reason for the discrepancy is that the Pd-H interactions in the model used here are purely repulsive, and hence the $\mathrm{H}$ appears to prefer the octahedral site because it is further from the neighboring Pd atoms. This is in contrast to the picture in Ref. 27 which assumed an attractive $\mathrm{Pd}-\mathrm{H}$ interaction which resulted in contraction of the Pd atoms at the octahedral site. However, these qualitative arguments have not been tested using a more realistic simulation model.

\section{ACKNOWLEDGMENTS}

This work was supported by a grant from the National Science Foundation (Award No. 1106219). We would also like to acknowledge helpful correspondence from Professor Pawel Keblinski, who let us review, before publication, results that demonstrate the correct method for computing partial enthalpy in the context of Green-Kubo thermal conductivity calculations.

${ }^{1}$ R. E. Howard and A. B. Lidiard, "Matter transport in solids," Rep. Prog. Phys. 27, 161 (1964).

${ }^{2}$ A. B. Lidiard, Handb. d. Phys. 20, 246 (1957) (Springer Verlag, Berlin).

${ }^{3}$ M. S. Green, "Markoff random processes and the statistical mechanics of time-dependent phenomena.2. Irreversible processes in fluids," J. Chem. Phys. 22, 398 (1954).

${ }^{4} \mathrm{R}$. Kubo, "Statistical-mehanical theory of irreversible processes. 1. General theory and simple applications to magnetic and conduction problems," J. Phys. Soc. Jpn. 12, 570 (1957).

${ }^{5}$ M. J. Gillan, "A simulation model for hydrogen in palladium: I. Singleparticle dynamics," J. Phys. C. 19, 6169 (1986).

${ }^{6} \mathrm{M}$. J. Gillan, "A simulation model for hydrogen in palladium: II. Mobility and thermotransport," J. Phys. C. 20, 521 (1986).

${ }^{7}$ D. J. Evans, "Homogeneous NEMD algorithm for thermal conductivityApplication of non-canonical linear response theory," Phys. Lett. A 91, 457 (1982).

${ }^{8}$ Z. McDargh and P. K. Schelling, "Molecular-dynamics approach for determining the vacancy heat of transport," Comput. Mater. Sci. 50, 2363 (2011).

${ }^{9}$ L. Onsager, "Reciprocal relations in irreversible processes. I.," Phys. Rev. 37, 405 (1931).

${ }^{10} \mathrm{P}$. Jund and R. Jullien, "Molecular-dynamics calculation of the the thermal conductivity of vitreous silica," Phys. Rev. B 59, 13707 (1999).

${ }^{11}$ J. H. Irving and J. G. Kirkwood, "The statistical mechanical theory of transport processes. IV. The equations of hydrodynamics," J. Chem. Phys. 18, 817 (1950).

${ }^{12}$ M. Parrinello and A. Rahman, "Polymorphic transitions in single crystals: A new molecular dynamics method," J. Appl. Phys. 52, 7182 (1981).

${ }^{13}$ R. Vogelsang and C. Hoheisel, "Thermal conductivity of a binary-liquid mixture studied by molecular dynamics with use of Lennard-Jones potentials," Phys. Rev. A 35, 3487 (1987).

${ }^{14}$ R. Vogelsang, C. Hoheisel, G. V. Paolini, and G. Ciccoti, "Soret coefficient of isotopic Lennard-Jones mixtures and the $\mathrm{Ar}-\mathrm{Kr}$ system as determined by equilibrium molecular-dynamics simulation," Phys. Rev. A 36, 3964 (1987).

${ }^{15}$ H. Babaei, P. Keblinski, and J. M. Khodadadi, "Equilibrium molecular dynamics determination of thermal conductivity for multi-component systems," J. Appl. Phys. 112, 054310 (2012).

${ }^{16}$ P. K. Schelling, S. R. Phillpot, and P. Keblinski, "Comparison of atomiclevel simulation methods for computing thermal conductivity," Phys. Rev. B 65, 144306 (2002).

${ }^{17}$ X. W. Zhou, S. Aubry, R. E. Jones, A. Greenstein, and P. K. Schelling, "Towards more accurate molecular dynamics calculation of thermal conductivity: Case study of GaN bulk crystals," Phys. Rev. B 79, 115201 (2009).

${ }^{18}$ D. P. Sellan, E. S. Landry, J. E. Turney, A. J. H. McGaughey, and C. H. Amon, "Size effects in molecular dynamics thermal conductivity predictions," Phys. Rev. B 81, 214305 (2010).

${ }^{19}$ M. J. Gillan, "The heat of transport in solids: A new theoretical approach," J. Phys. C 10, 1641 (1977). 
${ }^{20}$ M. J. Gillan, "Heat of transport in solids: II. Harmonic theory for a vacancy in the simple cubic lattice," J. Phys. C. 10, 3051 (1977).

${ }^{21}$ M. J. Gillan and M. W. Finnis, "Heat of transport in solids: III. Computer simulation of a vacancy in an fcc crystal," J. Phys. C. 11, 4469 (1978).

${ }^{22}$ C. Jones, P. J. Grout, and A. B. Lidiard, "The heat of transport of vacancies in solid argon," Philos. Mag. Lett. 74, 217 (1996).

${ }^{23}$ C. Jones, P. J. Grout, and A. B. Lidiard, "The heat of transport of vacancies in model fcc solids," Philos. Mag. A 79, 2051 (1999).
${ }^{24}$ P. J. Grout and A. B. Lidiard, "Computation of heats of transport in crystalline solids: II,” J. Phys. Condens. Matter 20, 425201 (2008).

${ }^{25}$ K. A. M. Dickens, P. J. Grout, and A. B. Lidiard, "Computation of heats of transport of vacancies in model crystalline solids: III,’ J. Phys. Condens. Matter 23, 265401 (2011).

${ }^{26}$ W. A. Oates and J. G. Shaw, "Thermomigration of hydrogen in palladium," Metall. Trans. 1, 3237 (1970).

${ }^{27}$ R. J. Asaro, D. Farkas, and Y. Kulkarni, "The Soret effect in diffusion in crystals," Acta Mater. 56, 1243 (2008). 